Article

\title{
Treatment with Pulsed Extremely Low Frequency Electromagnetic Field (PELF-EMF) Exhibit Anti-Inflammatory and Neuroprotective Effect in Compression Spinal Cord Injury Model
}

\author{
Yona Goldshmit ${ }^{1,2}$, Moshe Shalom ${ }^{1}$ and Angela Ruban ${ }^{1,3, *(\mathbb{D})}$ \\ 1 Steyer School of Health Professions, Sackler Faculty of Medicine, Tel-Aviv University, Tel Aviv 6997801, Israel; \\ yona.goldshmit@tauex.tau.ac.il (Y.G.); mosheshalom@mail.tau.ac.il (M.S.) \\ 2 Australian Regenerative Medicine Institute, Monash Biotechnology, 15 Innovation Walk, \\ Melbourne 3800, Australia \\ 3 Sagol School of Neuroscience, Tel Aviv University, Tel Aviv 6997801, Israel \\ * Correspondence: angellruban@tauex.tau.ac.il
}

Citation: Goldshmit, Y.; Shalom, M.; Ruban, A. Treatment with Pulsed

Extremely Low Frequency

Electromagnetic Field (PELF-EMF)

Exhibit Anti-Inflammatory and

Neuroprotective Effect in

Compression Spinal Cord Injury

Model. Biomedicines 2022, 10, 325 .

https://doi.org/10.3390/

biomedicines 10020325

Academic Editors: Yongdoo Choi and Pietro Veglianese

Received: 17 December 2021

Accepted: 26 January 2022

Published: 29 January 2022

Publisher's Note: MDPI stays neutral with regard to jurisdictional claims in published maps and institutional affiliations.

Copyright: (c) 2022 by the authors. Licensee MDPI, Basel, Switzerland. This article is an open access article distributed under the terms and conditions of the Creative Commons Attribution (CC BY) license (https:// creativecommons.org/licenses/by/ $4.0 /)$.

\begin{abstract}
Background: Spinal cord injury (SCI) pathology includes both primary and secondary events. The primary injury includes the original traumatic event, and the secondary injury, beginning immediately after the initial injury, involves progressive neuroinflammation, neuronal excitotoxicity, gliosis, and degeneration. Currently, there is no effective neuroprotective treatment for SCI. However, an accumulating body of data suggests that PELF-EMF has beneficial therapeutic effects on neurotrauma. The purpose of this study was to test the efficacy of the PELF-EMF SEQEX device using a compression SCI mouse model. Methods: C57BL/ 6 mice were exposed to PELF-EMF for $4 \mathrm{~h}$ on a daily basis for two months, beginning $2 \mathrm{~h}$ after a mild-moderate compression SCI. Results: The PELF-EMF treatment significantly diminished inflammatory cell infiltration and astrocyte activation by reducing Iba1, F4/80, CD68+ cells, and GAFP at the lesion borders, and increased pro-survival signaling, such as BDNF, on the neuronal cells. Moreover, the treatment exhibited a neuroprotective effect by reducing the demyelination of the axons of the white matter at the lesion's center. Conclusions: Treatment with SEQEX demonstrated significant anti-inflammatory and neuroprotective effects. Considering our results, this safe and effective rehabilitative device, already available on the market, may provide a major therapeutic asset in the treatment of SCI.
\end{abstract}

Keywords: spinal cord injury; electromagnetic field; neuroprotection; treatment; rehabilitation

\section{Introduction}

Spinal cord injury (SCI) is a traumatic event that results in altered sensory and motor function that can ultimately affect a patient's physical, psychological, and social wellbeing [1]. Statistics show that more than 27 million people worldwide suffer from longterm disabilities due to spinal cord injuries, $90 \%$ of which are due to trauma [2]. The life expectancy of SCI patients highly depends on the level of injury and the degree of preserved functions. Today, the estimated lifetime cost of a SCI patient is \$2.35 million [3]. Although there are extensive efforts underway to develop novel treatments for SCI, the current clinical treatment remains far from ideal [4,5]. Therapy for SCI is not adequately effective and consists mostly of hemodynamic stabilization, spinal cord decompression, and early rehabilitation [6]. Patient outcome is largely determined by the management of resultant symptoms and rehabilitation to maximize neural function [7]; however, rehabilitation is a long process that requires full patient cooperation [8,9]. It is therefore critical to explore and develop new rehabilitative interventions to improve the patients' neurological and functional outcomes. 
The pathophysiology of SCI is multilayered, including primary and secondary events. The primary injury results from traumatic mechanical force, producing diffuse or focal pathology. This initial phase is characterized by tissue deformation, membrane depolarization, disruption of blood vessels and axons, ischemia, and cell membrane damage $[10,11]$. The secondary injury begins within minutes of the initial injury and continues for months to years [7]. This secondary injury is triggered in response to the primary damage and leads to progressive neuroinflammation, neuronal excitotoxicity, axonal demyelination and degeneration, and ultimately, neuronal cell death [11-14]. While therapeutic efforts are often focused on the axon regeneration of the central nervous system (CNS), astrocytic and glial secondary responses significantly potentiate SCI damage [14,15]. During the first minutes following injury, microglial activation and monocyte/macrophage infiltration initiates into the CNS, and their numbers remain high for several months following injury $[11,16,17]$. Activated microglia/macrophages clear tissue debris and are part of the body's response to trauma [18]. Their prolonged activation, however, contributes to the secondary damage, or to the CNS injury, by releasing pro-inflammatory cytokines, neurotoxic factors, and reactive oxygen species that induce neurodegeneration at the injury site $[19,20]$. In addition to this, astrocytes react acutely to the CNS injury by increasing cytokine and chemokine production [21]. The results from animal studies suggest that limiting pro-inflammatory damage can improve SCI outcomes [22-24].

Few studies have examined the effects of using extremely low frequency electromagnetic field (ELF-EMF) in SCI and stroke models, but its use has demonstrated decreased neuronal/axonal degeneration and scar formation, increased neuronal plasticity, and improved functional recovery in animal models [25-27]. Exposing rats with SCI to PELF-EMF stimulation $(\mathrm{F}=50 \mathrm{~Hz}, \mathrm{Mf}=1 \mathrm{mT})$ for $4 \mathrm{~h}$ a day decreased the levels of TNF- $\alpha$ and IL-6, promoted remyelination, and increased the expression of BDNF [25]. In an ischemic stroke animal model, ELF-EMF treatment $(F=15.72 \mathrm{~Hz}, \mathrm{Mf}=10 \mu \mathrm{T})$ two days after occlusion increased neuronal regeneration and the expression of nestin and doublecortin (DCX) [26]. In a similar study, treatment $(\mathrm{F}=60 \mathrm{~Hz}, \mathrm{Mf}=10 \mathrm{mT})$ started within 30-40 min post occlusion decreased IL-1 $\beta$ and MMP9 levels and modulated the apoptotic cascade by promoting activation of the BDNF/TrkB/Akt signaling pathway, thus decreasing the expression of caspase-3 [27]. Furthermore, it has been reported that ELF-EMF exposure induces the early expression of neuronal differentiation markers, likely due to the opening of the L-type voltage-gated calcium channels via increased $\mathrm{Ca}^{2+}$ ions [28]. It has also been demonstrated that low frequency electromagnetic pulses reduced the IL-1 $\beta$ and TNF- $\alpha$ in nucleus pulposus cells and downregulate TNF- $\alpha$ as well as its transcription factor, nuclear factor kappa $\mathrm{B}(\mathrm{NFkB})$, in murine macrophages [29]. ELF-EMF-induced membrane depolarization has been shown to promote neuronal survival [30], probably through the above mechanisms.

The knowledge about the importance of early rehabilitation and the current lack of effective neuroprotective therapy brings us to examine an available and simple-to-use PELF-EMF device as a potential rehabilitative treatment in the SCI mouse model for mild to moderate compression injuries.

\section{Materials and Methods}

\subsection{Mice}

Male and female C57BL/ 6 adult mice (two months) were used in this study. We used young adult mice, as about $50 \%$ of spinal cord injuries occur in young people between the ages of 16 and 30 years. All experiments were conducted according to the Guidelines for the Use of Experimental Animals of the European Community and were approved by the Animal Care Committee of Tel Aviv University.

\subsection{Spinal Cord Compression}

All mice (25-35 g) were anesthetized by intraperitoneal injections of ketamine $(60 \mathrm{mg} / \mathrm{kg})$ and xylazine $(10 \mathrm{mg} / \mathrm{kg})$. The skin was shaved and wiped with $70 \%$ ethanol prior to making a longitudinal cut through the dorsal surface. Muscles and the laminal arches of thoracic 
vertebrae T8-T12 were removed, and the spinal cord was exposed. Calibrated Dumont \#5 forceps (with a spacer of $0.3 \mathrm{~mm}$ ) were placed in approximately the middle of the exposed segment of the spinal cord at the level of T10. The arms of the forceps were placed within the epidural space on adjacent sides, and their tips touched the floor of the vertebral canal in order to generate reproducible injuries. The spinal cord was then compressed until the spacers connected, and the compression was held for a duration of $5 \mathrm{~s}$. The skin was then joined with biological glue (M3 VetBond). Following the surgery, all mice received analgesia and antibiotics for 3 days post operation $(100 \mu \mathrm{L}$ subcutaneous injection of $1.2 \mathrm{mg} / \mathrm{mL}$ Carprieve and Baytril).

\subsection{PELF-EMF}

A SEQEX ${ }^{\circledR}$ device produced by S.I.S.T.E.M.I. S.R.L. (Trento, Italy), certified CSQ ISO13485, was used for the present study. This device produces complex pulsed electromagnetic fields using an analogue mechanism with a frequency range of 1 to $80 \mathrm{~Hz}$ and intensities ranging from 1 to $20 \mu \mathrm{T}$. The electromagnetic field produced by the device control unit (on which the electromagnetic field parameters are set) is emitted from a mat containing a Helmholtz coil that generates the pulsed extremely low frequency electromagnetic field (PELF-EMF)

\subsection{Treatment Protocol}

The mice were treated with PELF-EMF for 7 weeks, 5 times a week, for $4 \mathrm{~h}$. Each treatment consisted of 4 cycles of $1 \mathrm{~h}$ duration (Table 1 ), with treatments starting two hours following compression.

Table 1. The PELF-EMF 9-cycle treatment protocol.

\begin{tabular}{cccccc}
\hline Step & Frequency $\mathbf{( H z )}$ & Field Intensity $(\boldsymbol{\mu} \mathbf{T})$ & T-On $(\mathbf{m i n})$ & T-Off $(\mathbf{m i n})$ & Duration $(\mathbf{m i n})$ \\
\hline 1 & 15 & 20 & 2 & 3 & 14 \\
2 & 75 & 20 & 4 & 1 & 12 \\
3 & 2 & 20 & 2 & 3 & 14 \\
4 & 20 & 20 & 3 & 1 & 14 \\
5 & 12 & 20 & 3 & 1 & 14 \\
6 & 3 & 20 & 2 & 1 & 12 \\
7 & 50 & 20 & 5 & 2 & 14 \\
8 & 25 & 20 & 5 & 1 & \\
9 & 10 & & & & \\
\hline
\end{tabular}

The PELF-EMF protocol included the use of complex modulation using different frequencies. The combinations, referred to as steps, are modulated in order to produce the PEMF for a given time (T-on), followed by a period without emission (T-off) for a blocked time (duration). Each frequency has been chosen for the PELF-EMF protocol based on significant experimental results reported previously:

(a) Neuroprotection by the reduction of Glu+-induced excitotoxicity, step $1(15 \mathrm{~Hz})[31,32]$.

(b) Modulation of local inflammation in order to increase the anti-inflammatory effect, steps 2,3 , and $6(75 \mathrm{~Hz}$ and $2 \mathrm{~Hz})[25,33]$.

(c) Improvement of the removal of Glu+ from the damaged area (ion cyclotron resonance hypothesis), steps 4 and 5 [34].

(d) Stimulation of the recovered area after SCI, step $7(50 \mathrm{~Hz}$, which also has an antiinflammatory effect), and neuro-regeneration, steps 8 and 9 (25 Hz and $10 \mathrm{~Hz})$ [25,35-37].

\subsection{Immunohistochemistry}

Cryostat longitudinal sections $(20 \mu \mathrm{m})$ of fixed frozen tissue were stained using standard immunohistochemistry procedures. Primary antibodies included rabbit anti-GFAP (1:1000, Dako, Copenhagen, Denmark); rabbit anti-Iba1 (1:400; Abcam, Zotal Ltd., Tel AvivYafo, Israel); rat anti-CD68 (1:500; Thermo-Fisher Science, Qiryat Shemona, Israel); rat anti-F4/80 (1:800; Abcam, Zotal Ltd.); mouse anti-ßiii tubulin (1:1000; Promega, Kibbutz Beit Ha'Emek, Israel); rabbit anti-BDNF (1:200; Abcam, Zotal Ltd.); rabbit anti-MBP 
(1:500; Abcam, Zotal Ltd.); and mouse anti-NeuN (1:500; Millipore, Sigma-Aldrich Ltd., Rehovot, Israel). Secondary antibodies included goat anti-rat or rabbit Alexa Fluor 488 and goat anti-mouse Alexa 546 1:1000 (Invitrogen, Sigma-Aldrich Ltd., Rehovot, Israel). Nuclei were visualized using DAPI (Sigma-Aldrich Ltd., Rehovot, Israel)). To obtain the immunofluorescence density of different antibody markers, a series of 20- $\mu$ m-thick longitudinal sections were cut. For each measurement, sections were taken at $200-\mu \mathrm{m}$ intervals, and sectioned tissue included both white and grey matter (15 sections per animal for each marker, $n=10$ /group). DAPI immunofluorescence staining was used to define the edge of the lesion and the size was calculated and compared to the vehicle-treated control SCI mice. All measurements were performed using ImageJ software.

\subsection{Histological Analysis}

Longitudinal sections ( $20 \mu \mathrm{m} ; 500-\mu \mathrm{m}$ intervals, 8 sections per animal; $n=10)$ of fixed, frozen mice spinal cord tissue were stained at 8 weeks post-SCI. They were stained with Cresyl Violet eosin (Sigma-Aldrich Ltd., Rehovot, Israel) for lesion area assessment and Luxol Fast Blue (LFB; Sigma-Aldrich Ltd., Rehovot, Israel) for white matter sparing analysis. The center of each lesion was defined as the section containing the least amount of spared white matter. LFB-positive myelinated areas were measured at the epicentre, and different distances, rostral and caudal from the epicentre, were recorded as specified.

Sections were imaged by fluorescence microscopy using an Olympus IX83 fluorescence microscope, an ORCA digital camera, and cellSens Dimension Version 3; images were sized using Adobe Photoshop 11 and Illustrator 14. All fluorescence density or intensity measurements were performed using ImageJ software.

\subsection{Behavioral Analyses}

Open field locomotion score: Mice were evaluated for $3 \mathrm{~min}$ using the modified Basso-Beattie-Bresnahan (mBBB) 9-point scoring system (control $n=10$, EMF $n=10$ ).

\subsection{CatWalk}

Gait measures were determined using the CatWalk XT 10.6 system5 (Noldus Information Technology, Wageningen, The Netherlands) three times during the experiment: 1 week post injury, 5 weeks post injury, and 7 weeks post injury. It is important to note that we encouraged all mice on the platform to walk at their maximum speed by inflating compressed air on their backs to reduce the variability in running speeds between animals. The visual data were digitized and analyzed using CatWalk XT for static and dynamic gait kinematics, using distance, time, and intensity differences between hind paw prints as measures contributing to gait. Each mouse was placed on the platform and permitted to cross the walking path for at least three compliant runs, when possible (not all mice were cooperative, and some walked less than others), as detected by the CatWalk XT system. Three variables were chosen based on their relevance to human locomotion and human SCI: the base of support (the width, in $\mathrm{cm}$, between the two hind paws), the stride length (the distance, in $\mathrm{cm}$, between subsequent placements of the left hind paw), and the swing speed (speed (distance unit/second) of the paw during swing).

\subsection{Statistical Analysis}

All statistical analyses were conducted using the GraphPad Prism Program, Version 5.03, for Windows. The significance between the treated and untreated groups was evaluated using the two-tailed $t$-test with $95 \%$ confidence when comparing two parameters in the data presented in Figures $1-4\left({ }^{*} p<0.05,{ }^{* *} p<0.01,{ }^{* * *} p<0.001\right)$. The non-parametric Mann-Whitney U test was used to assess the significance of the differences in the CatWalk behavioral analysis (Figure 5; $p<0.05$ ). Data are expressed as mean \pm standard error of the mean (SEM), or standard deviation (STDEV), as indicated in the figure legends. 

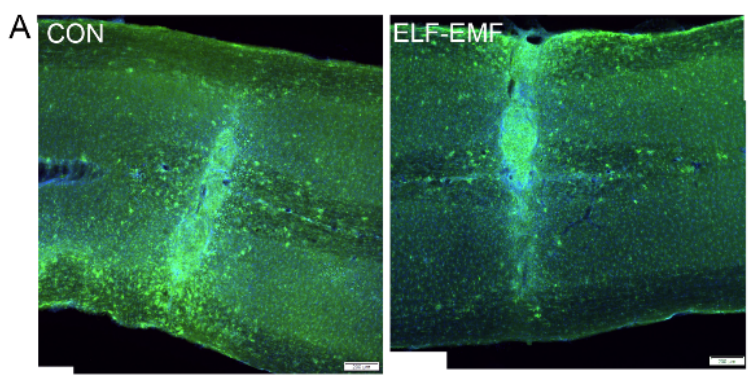

|ba1/DAP|

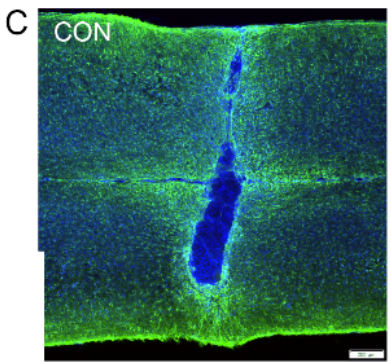

GFAP/DAPI

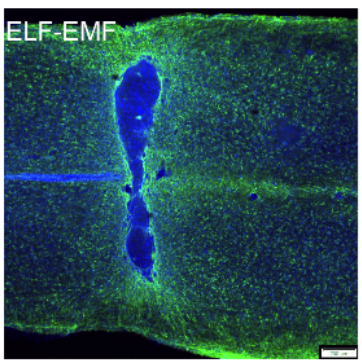

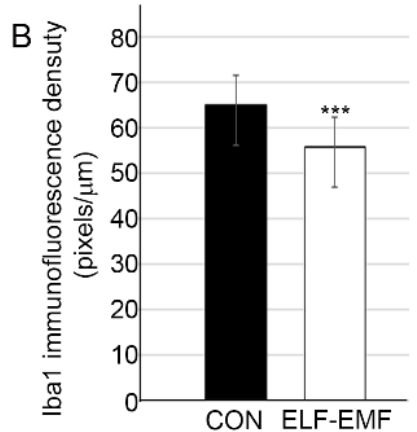

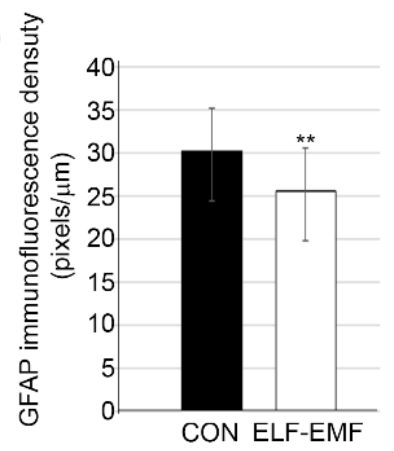

Figure 1. Decreased microglia activation and astrogliosis in PELF-EMF-treated mice after SCI. Two weeks after SCI (A) Representative images of the lesion site of Iba1 (green) immunostaining; DAPI (blue) demonstrate the lesion site. The scale bar is $200 \mu \mathrm{m}$. (B) Quantitation of Iba1 at the lesion site shows a significant decrease in the PELF-EMF-treated compared to the non-treated mice $(n=8$ in each group; ${ }^{* * *} p<0.001$ ). (C) Representative images of the lesion site of GFAP (green) immunostaining; DAPI (blue) demonstrate the lesion site. The scale bar is $200 \mu \mathrm{m}$. (D) Quantitation of GFAP at the lesion site shows a significant decrease in the PELF-EMF-treated compared to the non-treated mice $\left(n=8\right.$ in each group; $\left.{ }^{* *} p<0.01\right)$.

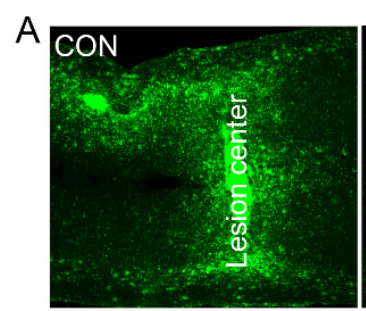

CD68

\section{C}

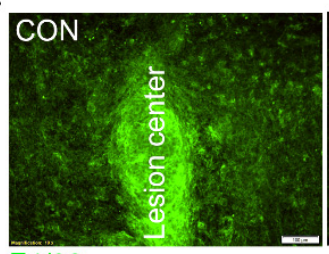

$\mathrm{F} 4 / 80$
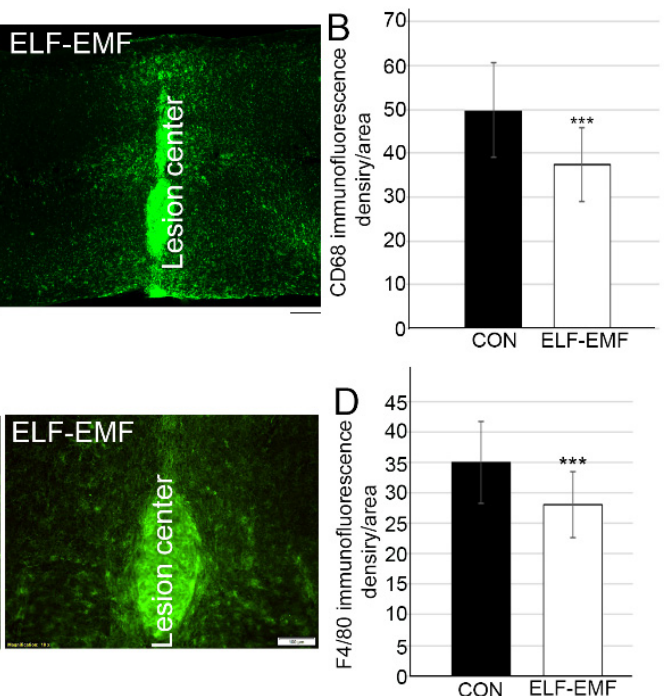

Figure 2. Decrease of other microglia activation markers in PELF-EMF-treated mice after SCI. Two weeks after SCI (A) Representative images of the lesion site of CD68 (green) immunostaining; DAPI (blue) demonstrate the lesion site. The scale bar is $100 \mu \mathrm{m}$. (B) Quantitation of CD68 at the lesion site shows a significant decrease in the PELF-EMF-treated compared to the non-treated mice. The results are mean $\pm \mathrm{SD}\left(n=8 /\right.$ group; $\left.^{* * *} p<0.001\right)$. (C) Representative images of the lesion site of F4/80 (green) immunostaining; DAPI (blue) demonstrate the lesion site. The scale bar is $100 \mu \mathrm{m}$. (D) Quantitation of F4/80 at the lesion site shows a significant decrease in the PELF-EMF-treated compared to the non-treated mice. The results are mean $\pm \operatorname{SD}\left(n=8\right.$ in each group; $\left.{ }^{* * *} p<0.001\right)$. 

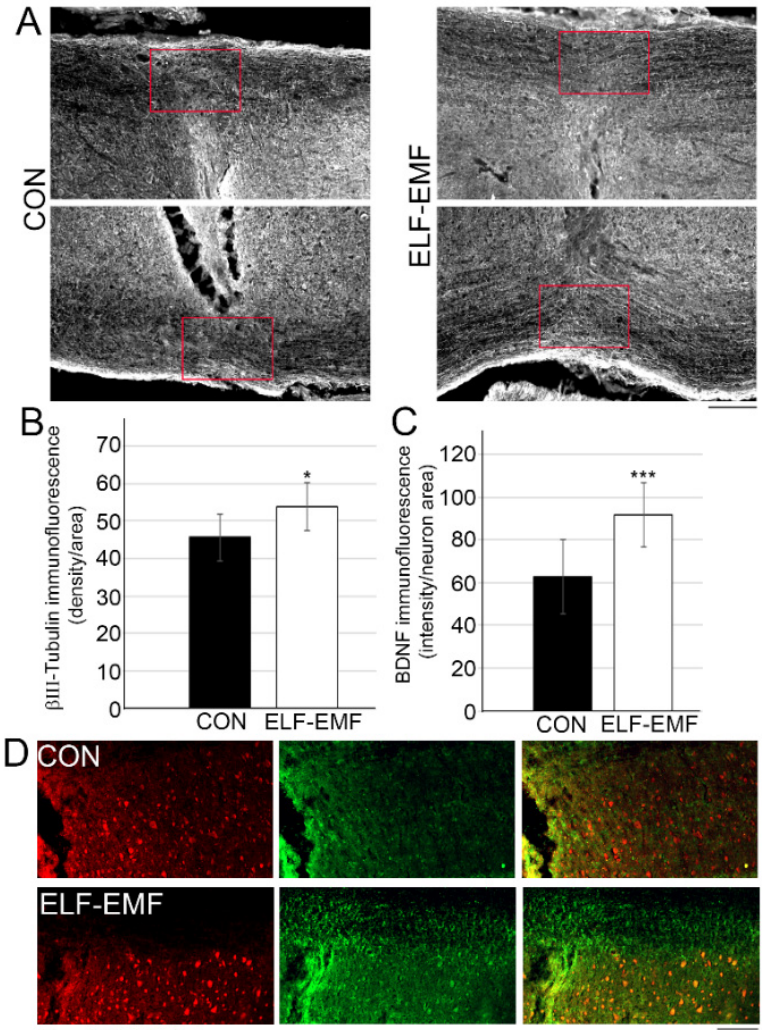

NeuN/BDNF

Figure 3. The increased axonal survival and BDNF expression in PELF-EMF-treated mice after SCI. Two months after SCI (A) Representative images of the white matter at the lesion site of the $\beta I I I-t u b u l i n$ immunostaining; the scale bar is $100 \mu \mathrm{m}$. (B) Quantitation of the $\beta$ III-tubulin density immunostaining at the lesion site shows a significant increase in the PELF-EMF-treated compared to the non-treated mice. The results are mean \pm SD (the red box represents the area of analysis; $n=10$ in each group; $\left.{ }^{*} p<0.05\right)$. (C) Quantitation of the BDNF (green) expression density in neuronal cells (NeuN in red) close to the lesion site shows a significant increase in the PELF-EMF-treated compared to the non-treated mice. The results are mean $\pm \operatorname{SD}\left(n=10\right.$ in each group; $\left.{ }^{* * *} p<0.001\right)$. (D) Representative images of lesion site of the BDNF (green) and NeuN (red) immunostaining; DAPI (blue) demonstrate the lesion site. The scale bar is $100 \mu \mathrm{m}$. 
A Control
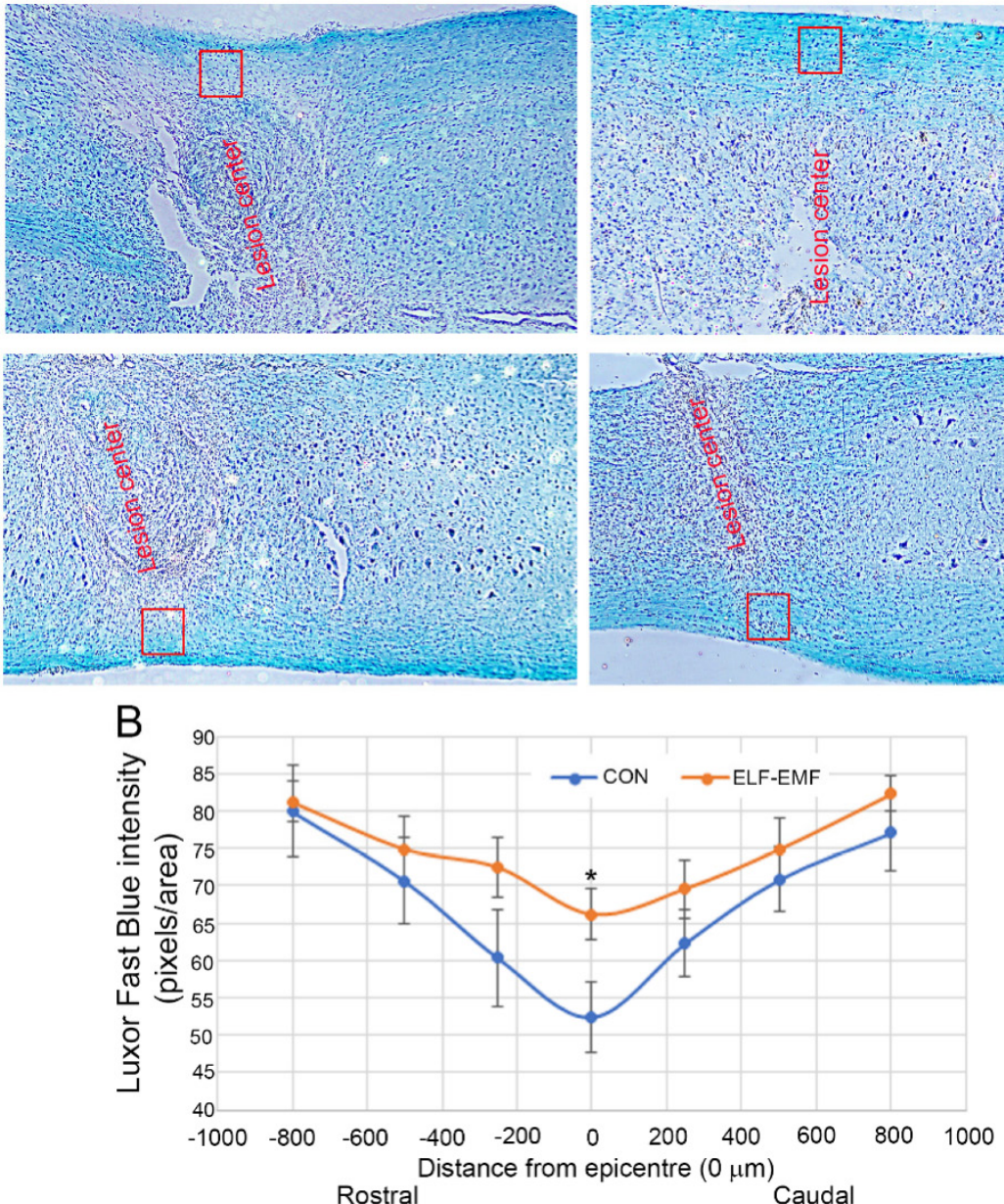

Caudal
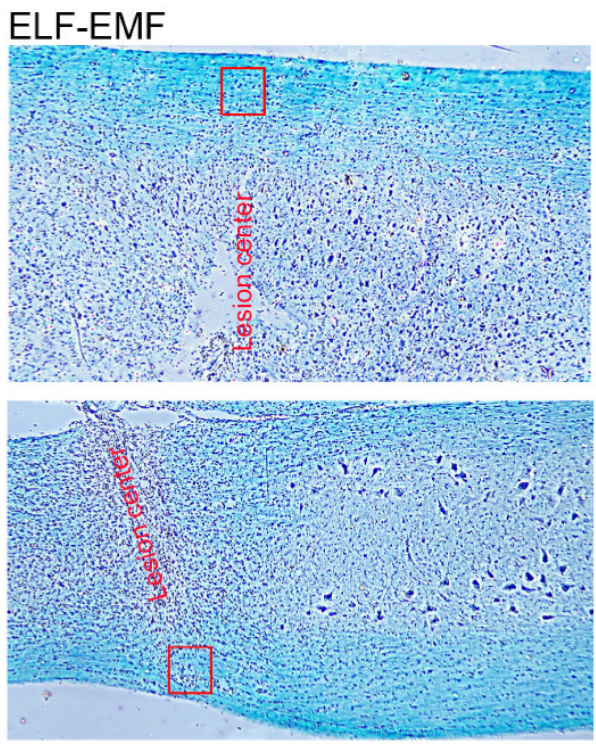

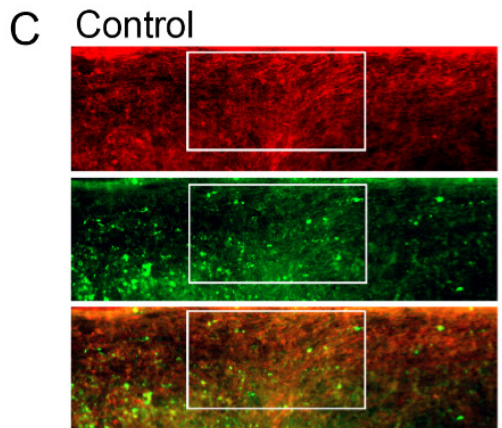

Blll-Tubulin/MBP
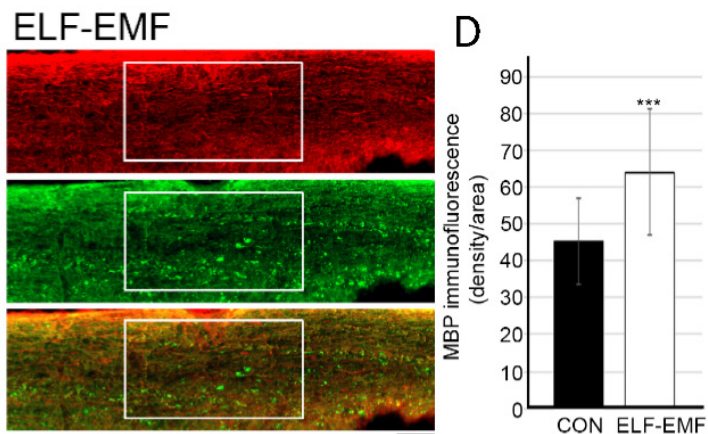

Figure 4. The reduced axonal demyelination and increased MBP expression in PELF-EMF-treated mice after SCI. Two months after SCI (A) Representative images both sides of the white matter of the spinal cord stained with LFB. The injury epicentre (0) is marked in the red box. The scale bar is $100 \mu \mathrm{m}$. (B) Quantitative analysis of residual myelin in different rostral and caudal distances from the epicentre of the white matter in the PELF-EMF and the control treated groups shows a significant decrease in demyelination in the PELF-EMF-treated mice. Data represent the mean \pm SD ( $n=10$ /group; $\left.{ }^{*} p<0.05\right)$. (C) Representative images of the white matter at the lesion site with MBP immunostaining; the scale bar is $100 \mu \mathrm{m}$. (D) Quantitation of the MBP density immunostaining at the lesion site shows a significant increase in the PELF-EMF-treated compared to the non-treated mice. Results are mean $\pm \mathrm{SD}\left(n=10\right.$ group; $\left.^{* * *} p<0.001\right)$. 

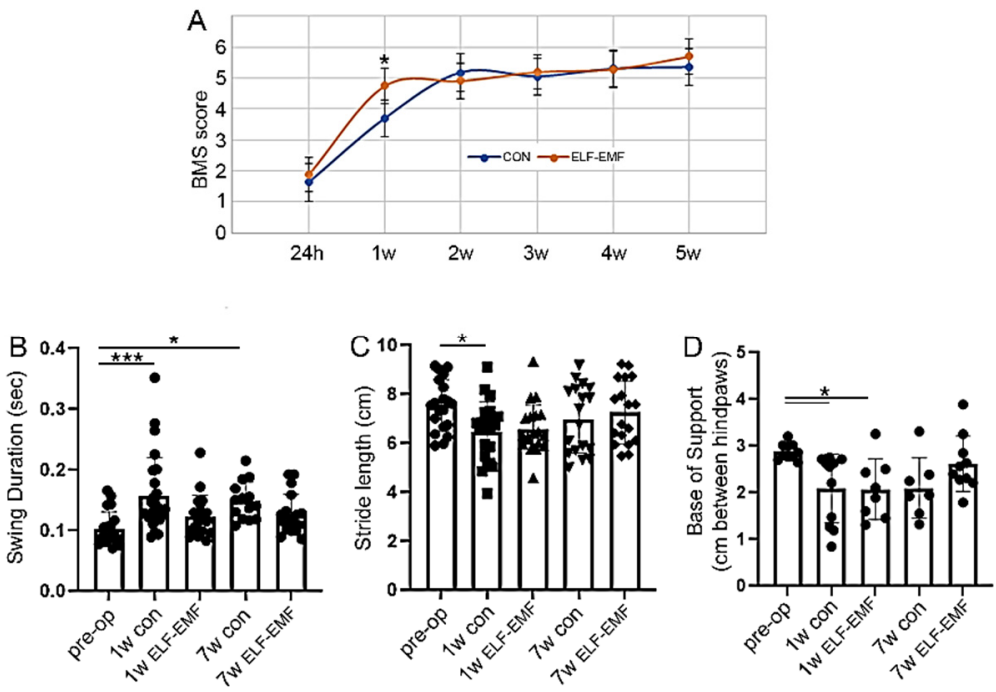

Figure 5. The improved motor function in the PELF-EMF-treated mice after SCI. Two months after $\mathrm{SCI}$, the motor function recovery of the mice was assessed using different behavioral tests. (A) mBBB scores demonstrated a significant improvement in the first week after SCI in the PELF-EMF-treated mice compared to the control; the subsequent weeks showed no difference between the groups ( $n=10$ animals/group. The results are the mean \pm SEM ( $*<0.05$; non-parametric Mann-Whitney test, $\alpha$ set to $5 \%$ ). The CatWalk analysis of swing duration (B), stride length (C), and the base of support (D) ( $n=10$ animals/group); the data are expressed as the mean \pm SEM, one-way ANOVA followed by Bonferroni's multiple comparison test; ${ }^{*} p<0.05$, ${ }^{* * *} p<0.001$ ).

\section{Results}

Since in our previous study, we demonstrated the anti-inflammatory effect of the reduction in glutamate excitotoxicity in spinal cord injured mice, we selected a $15 \mathrm{~Hz}$ frequency for use in step 1 [38]. This frequency has shown an anti-glutamatergic effect and a reduction in glutamate levels in vitro [31,32]. In addition, the $2 \mathrm{~Hz}$ and $75 \mathrm{~Hz}$ frequencies demonstrated a change in local inflammation in the CNS and an increased anti-inflammatory response after spinal cord injury in the rat mode $[25,33]$ and were therefore used in steps 2,3, and 6 of the protocol. In steps 4 and 5, the $20 \mathrm{~Hz}$ and $12 \mathrm{~Hz}$ frequencies, respectively, were applied to improve the removal of Glu+ from the damaged area based on the Ion Cyclotron Resonance Hypothesis [34]. The $50 \mathrm{~Hz}$ frequency increased the recovery and the anti-inflammatory response after spinal cord injury in rats [25]. We used this frequency in step 7 of the protocol. The $25 \mathrm{~Hz}$ and $10 \mathrm{~Hz}$ frequencies showed neuroprotective and regenerative effects in the neuronal culture from ischemic brain tissues and promoted the restoration of sensorimotor functions in adult rats with a hemisection of the thoracic spinal cord $[36,37]$. These frequencies were used in steps 8 and 9.

\subsection{PELF-EMF Treatment Reduced Astrocyte and Microglia Reactivity}

In order to determine whether the PELF-EMF treatment reduced the pro-inflammatory environment two weeks following SCI compression, we examined the degree of activation of microglia/macrophages and astrocytes at the lesion site. Examination of the density of GFAP and Iba1 expression at the lesion site demonstrated that the PELF-EMF treatment significantly decreased glial scarring and inflammation, respectively, at and around the lesion site (Figure 1). Further examination of the M1 phenotype of the activated microglia/macrophages, using Cd68 and F4/80 markers [39,40] was performed. Significantly reduced microglia/macrophage activation was demonstrated by evaluation using CD68+ staining (control $49.9+10.9$; PELF-EMF $37.2+8.4 ;{ }^{* * *} p<0.001$ ) (Figure 2B). Interestingly, some CD68+ cells extended diffusely beyond the lesion cavity borders and glial interface into the grey and white matter in the control groups, compared to the results from the PELF-EMF treated groups. In the treated groups, CD68+ cells were more restricted to the 
lesion center (Figure 2A). Staining with F4/80, which preferentially stains macrophages and activated microglia, demonstrated positively stained cells mainly at the lesion site and mostly in the control group, with reduced quantified density in the PELF-EMF treated groups (Figure 2C,D).

\subsection{PELF-EMF Treatment Increases Axonal Survival and BDNF Expression at the Lesion Site}

Two months following daily PELF-AMF treatment, significantly higher $\beta$ III-tubulin levels were detected in the spinal cord's white matter at the lesion sites (Figure 3A,B), suggesting that the electromagnetic treatment led to axonal survival at the lesion area. We further examined whether this treatment facilitated pro-survival molecule expression [41]. BDNF, an important neurotrophic factor used in experimental neurotrauma treatments to promote neurogenesis, neuroprotection, axonal sprouting, myelination, and synaptic plasticity, was significantly increased in neuronal cells adjacent to the lesion site in the PELF-EMF group compared to the control group (control 62.7+ 17.3; PELF-EMF 91.6 + 15.1; *** $p<0.001$ ) as shown in Figure 3C,D.

\subsection{PELF-EMF Treatment Moderated the Area of Demyelination at the Lesion Site}

BDNF is known to play a role in myelin structure formation, maintenance, and repair, and has been suggested to be a critical factor involved in remyelination and/or structural repair of myelin after neurotrauma [42,43]. Luxol Fast Blue (LFB) histological staining of longitudinal sections at the injury site revealed enhanced myelin sparing in the PELFEMF-treated mice compared with the untreated mice (Figure 4A,B). Significant myelin loss prevention in the PELF-EMF mice was evident only in the white matter of the lesion center, up to $250 \mu \mathrm{m}$ rostral and caudal to the injury. Additional analysis of myelin sparing was examined using immunostaining of the myelin binding protein (MBP) in the white matter at the center of the lesion (Figure 4C) and it was found that the MBP density was significantly reduced in the control group when compared to the PELF-EMF-treated group (control 45.2 + 11.7; ELF-EMF $64.16+17.08 ;{ }^{* * *} p<0.001$ ). These results may suggest that the electromagnetic treatment reduced the demyelination of the axons through inhibition of the inflammatory response and enhancement of the pro-survival factor secretion.

\subsection{ELF-EMF Treatment Reduced Functional Deficits a Week after the Injury}

As shown in Figure 5, the PELF-EMF-treated mice displayed significant improvement in locomotor recovery at seven days post SCI, but this significance was lost at 14 days after the injury. There was a significant improvement in the mBBB score at 7 days post injury, and the score remained the same up to 5 weeks post-injury (Figure 5A). Mice in the control group reached the same score as the PELF-EMF treated mice at week two, but had significantly lower mBBB scores at one week post-injury.

Similar results were obtained using CatWalk gait analysis. Compared with mice prior to injury, the control group showed significantly lower measurements in swing duration and stride length compare to the PELF-EMF-treated mice, which demonstrated better locomotive abilities (Figure 5B,C). Poorer swing duration measures continued in the control group, even at 7 weeks post-injury. In both groups, the base of support was significantly reduced, compared to that in the pre-operative stage (Figure 5D). These results may suggest that the PELF-EMF treatment produces greater improvement in motor abilities in the first week after injury when compared to control groups, possibly as a result of decreased inflammation.

\section{Discussion}

Over the past decade, an accumulating body of data suggests that PELF-EMF, in the frequency range of $<100 \mathrm{~Hz}$ and a field strength $<5 \mathrm{mT}$, exhibits beneficial therapeutic effects in the treatment of neurotrauma, without any adverse effects. Here we examined its benefit in a mild to moderate SCI model. 
In this study, we demonstrated that daily treatment with PELF-EMF, beginning within two hours after SCI, significantly diminished the pro-inflammatory response by reducing microglia and astroglia activation and increasing pro-survival and anti-apoptotic signaling, such as BDNF expression, in mice. Moreover, this treatment exhibited a neuroprotective effect by reducing the axon demyelination in the white matter of the lesion center. The antiinflammatory and neuroprotective effects of PELF-EMF treatment also improved functional performance during the first week post-injury; however, this improvement was weakened in subsequent weeks.

CNS injury is almost always accompanied by some degree of reactive gliosis, inflammation, and scarring [24,44]. Microglia are the first non-neuronal cells that become activated following neurotrauma, and they are the main source of pro-inflammatory mediators in the CNS. Activated astrocytes and microglia release a wide variety of cytokines, growth factors, and other inflammatory mediators, promoting axonal degeneration, demyelination, and scar formation [45]. Reactive astrocytes densely populate the borders of the injury epicenter, strongly upregulate the intermediate filament protein (such as GFAP) expression, and secrete chondroitin sulfate proteoglycans (CSPGs) into the extracellular matrix, organizing astrocytes into a barrier-like structure that inhibits neural sprouting through this area $[44,46,47]$. Attenuation of this early inflammatory response to spinal cord injury (SCI) may therefore limit the extent of the secondary tissue injury and, accordingly, the consequent disability [48]. In stroke patients, rehabilitative 4-week treatment with ELF-EMF $(\mathrm{F}=40 \mathrm{~Hz}, \mathrm{Bm}=5 \mathrm{mT})$, in combination with physiotherapy, reduced both IL-1 $\beta$ plasma and IL-1 $\beta$ mRNA expression levels and increased IL-2 plasma levels without any adverse effects [49]. In addition, no cytotoxic or genotoxic effects were detected in a human mesenchymal stromal cell exposed to an ELF-EMF of $5 \mathrm{~Hz}, 0.4 \mathrm{mT}$, for $20 \mathrm{~min} /$ day, $3 \mathrm{x} /$ week, for 2 weeks [50].

Here we demonstrated significantly reduced expression of GFAP at and around the lesion site after two weeks of daily treatment with PELF-EMF. Furthermore, the expression of M1 pro-inflammatory CD68+ microglia/macrophages was reduced and limited to the lesion site in the treated group, as compared to the significantly higher and more diffused expression in the untreated group. It is understood that shortly after neurotrauma, as a result of the primary injury, the predominantly M2-like microglia/macrophage environment shifts to an M1 pro-inflammatory type, promoting secondary damage [51-53]. It has been shown that the M1 microglia/macrophages indeed have a neurotoxic effect, while the anti-inflammatory M2 type promotes regenerative growth in response to CNS injury $[24,52,54]$. In our study, long-term neuroprotective markers such as BDNF, which has been identified as a potent promoter of neuronal cell survival and regeneration, were significantly elevated in the lesion site of the treated group two months post-injury. The elevated expression of BDNF, followed by the diminished loss of myelin, supports our hypothesis that the prevention of pro-inflammatory events shortly after spinal cord injury has a long-term neuroprotective effect. Nevertheless, motor function improved significantly in the first week only in the PELF-EMF treated mice, although later, the control group achieved the same status as the treatment group, likely because the injury was mild enough for mice from both groups to gain a high motor recovery score. We believe that the proposed rehabilitative treatment will be less effective in more severe cases of SCI. We could still see a trend of improvement in the gait of the PELF-EMF-treated mice in all the CatWalk parameters we presented in Figure 4 because the injuries to the mice were mild compression injuries, indicating that a combined treatment approach should be examined in the future for better neuro-functional outcomes in SCI patients.

\section{Conclusions}

To date, there is no FDA-approved treatment that can prevent the development of secondary SCI, nor one that induces regenerative processes [5]. Nonetheless, a moderate degree of functional recovery can be achieved using rehabilitative motor training $[38,55,56]$. Rehabilitation is probably one of the most important interventions following neurotrauma, 
and it can potentially improve a patient's quality of life significantly. However, multitrauma and the management of other medical complications in the acute post-injury setting often preclude or complicate early rehabilitation that demands the patient's active participation. Therefore, a simple, safe, and approved-for-use rehabilitation device that can be combined with traditional therapy, without the need to involve the patient in active participation immediately after a spinal cord injury, may have significant therapeutic value.

Author Contributions: Conceptualization, Y.G. and A.R.; methodology, Y.G. and A.R.; validation, Y.G. and A.R.; formal analysis, Y.G.; writing—original draft preparation, Y.G., M.S. and A.R.; writingreview and editing, Y.G., M.S. and A.R.; visualization, Y.G.; supervision, A.R. All authors have read and agreed to the published version of the manuscript.

Funding: We are very grateful to Dani Schaumann and Nessia Laniado for their generous donations supporting this research.

Institutional Review Board Statement: The study was conducted according to the guidelines of the Declaration of Helsinki, and approved by the Institutional Ethics Committee of Tel Aviv University (protocol code 01-20-008 and date of approval 15.01.2020).

Data Availability Statement: The data presented in this study are available on request from the corresponding author.

Acknowledgments: We thank S.I.S.T.E.M.I. S.R.L. (Trento, Italy) for providing the PELF-EMF device implemented in this project. We thank Alessandro Grego. for his professional help and support throughout the project.

Conflicts of Interest: The authors declare no conflict of interest. The funders had no role in the design of the study; the collection, analyses, or interpretation of data; the writing of the manuscript; or the decision to publish the results.

\section{References}

1. Jain, N.B.; Harris, M.B.; Garshick, E. Trends in Traumatic Spinal Cord Injury-Reply. JAMA 2015, 314, 1643-1644. [CrossRef] [PubMed]

2. GBD 2016 Traumatic Brain Injury and Spinal Cord Injury Collaborators. Global, regional, and national burden of traumatic brain injury and spinal cord injury, 1990-2016: A systematic analysis for the Global Burden of Disease Study 2016. Lancet Neurol. 2019, 18, 56-87. [CrossRef]

3. Hachem, L.D.; Ahuja, C.S.; Fehlings, M.G. Assessment and management of acute spinal cord injury: From point of injury to rehabilitation. J. Spinal Cord Med. 2017, 40, 665-675. [CrossRef] [PubMed]

4. Tran, A.P.; Silver, J. Systemically treating spinal cord injury. Science 2015, 348, 285-286. [CrossRef]

5. Karsy, M.; Hawryluk, G. Modern Medical Management of Spinal Cord Injury. Curr. Neurol. Neurosci. Rep. 2019, 19, 65. [CrossRef] [PubMed]

6. Huang, H.; Young, W.; Skaper, S.; Chen, L.; Moviglia, G.; Saberi, H.; Al-Zoubi, Z.; Sharma, H.S.; Muresanu, D. Clinical Neurorestorative Therapeutic Guidelines for Spinal Cord Injury (IANR/CANR version 2019). J. Orthop. Transl. 2020, $20,14-24$. [CrossRef]

7. Ahuja, C.S.; Wilson, J.R.; Nori, S.; Kotter, M.R.; Druschel, C.; Curt, A.; Fehlings, M.G. Traumatic spinal cord injury. Nat. Rev. Dis. Primers 2017, 3, 17018. [CrossRef]

8. Loy, K.; Schmalz, A.; Höche, T.; Jacobi, A.; Kreutzfeldt, M.; Merkler, D.; Bareyre, F.M. Enhanced Voluntary Exercise Improves Functional Recovery following Spinal Cord Injury by Impacting the Local Neuroglial Injury Response and Supporting the Rewiring of Supraspinal Circuits. J. Neurotrauma 2018, 35, 2904-2915. [CrossRef]

9. Elbasiouny, S.M.; Moroz, D.; Bakr, M.M.; Mushahwar, V.K. Management of spasticity after spinal cord injury: Current techniques and future directions. Neurorehabil. Neural Repair. 2010, 24, 23-33. [CrossRef]

10. Mortazavi, M.M.; Verma, K.; Harmon, O.A.; Griessenauer, C.J.; Adeeb, N.; Theodore, N.; Tubbs, R.S. The microanatomy of spinal cord injury: A review. Clin. Anat. 2015, 28, 27-36. [CrossRef]

11. Ahmed, A.; Patil, A.A.; Agrawal, D.K. Immunobiology of spinal cord injuries and potential therapeutic approaches. Mol. Cell. Biochem. 2018, 441, 181-189. [CrossRef] [PubMed]

12. Gensel, J.C.; Zhang, B. Macrophage activation and its role in repair and pathology after spinal cord injury. Brain Res. 2015, 1619, 1-11. [CrossRef]

13. Okada, S.; Hara, M.; Kobayakawa, K.; Matsumoto, Y.; Nakashima, Y. Astrocyte reactivity and astrogliosis after spinal cord injury. Neurosci. Res. 2018, 126, 39-43. [CrossRef] [PubMed] 
14. Beck, K.D.; Nguyen, H.X.; Galvan, M.D.; Salazar, D.L.; Woodruff, T.M.; Anderson, A.J. Quantitative analysis of cellular inflammation after traumatic spinal cord injury: Evidence for a multiphasic inflammatory response in the acute to chronic environment. Brain 2010, 133 Pt 2, 433-447. [CrossRef]

15. Shultz, R.B.; Zhong, Y. Minocycline targets multiple secondary injury mechanisms in traumatic spinal cord injury. Neural Regen. Res. 2017, 12, 702-713. [PubMed]

16. Prüss, H.; A Kopp, M.; Brommer, B.; Gatzemeier, N.; Laginha, I.; Dirnagl, U.; Schwab, J.M. Non-resolving aspects of acute inflammation after spinal cord injury (SCI): Indices and resolution plateau. Brain Pathol. 2011, 21, 652-660. [CrossRef] [PubMed]

17. Fleming, J.C.; Norenberg, M.D.; Ramsay, D.A.; Dekaban, G.A.; Marcillo, A.E.; Saenz, A.D.; Pasquale-Styles, M.; Dietrich, W.D.; Weaver, L.C. The cellular inflammatory response in human spinal cords after injury. Brain 2006, 129 Pt 12, 3249-3269. [CrossRef] [PubMed]

18. Anwar, M.A.; Al Shehabi, T.S.; Eid, A.H. Inflammogenesis of Secondary Spinal Cord Injury. Front. Cell. Neurosci. 2016, 10, 98. [CrossRef] [PubMed]

19. Liddelow, S.A.; Guttenplan, K.A.; Clarke, L.E.; Bennett, F.C.; Bohlen, C.J.; Schirmer, L.; Bennett, M.L.; Münch, A.E.; Chung, W.-S.; Peterson, T.C.; et al. Neurotoxic reactive astrocytes are induced by activated microglia. Nature 2017, 541, 481-487. [CrossRef]

20. Thuret, S.; Moon, L.D.; Gage, F.H. Therapeutic interventions after spinal cord injury. Nat. Rev. Neurosci. 2006, 7, 628-643. [CrossRef] [PubMed]

21. Pineau, I.; Sun, L.; Bastien, D.; Lacroix, S. Astrocytes initiate inflammation in the injured mouse spinal cord by promoting the entry of neutrophils and inflammatory monocytes in an IL-1 receptor/MyD88-dependent fashion. Brain Behav. Immun. 2010, 24, 540-553. [CrossRef] [PubMed]

22. Ramer, L.M.; Ramer, M.S.; Bradbury, E.J. Restoring function after spinal cord injury: Towards clinical translation of experimental strategies. Lancet Neurol. 2014, 13, 1241-1256. [CrossRef]

23. David, S.; Lopez-Vales, R.; Wee Yong, V. Harmful and beneficial effects of inflammation after spinal cord injury: Potential therapeutic implications. Handb. Clin. Neurol. 2012, 109, 485-502. [PubMed]

24. Orr, M.B.; Gensel, J.C. Spinal Cord Injury Scarring and Inflammation: Therapies Targeting Glial and Inflammatory Responses. Neurotherapeutics 2018, 15, 541-553. [CrossRef] [PubMed]

25. Li, Z.; Yao, F.; Cheng, L.; Cheng, W.; Qi, L.; Yu, S.; Zhang, L.; Zha, X.; Jing, J. Low frequency pulsed electromagnetic field promotes the recovery of neurological function after spinal cord injury in rats. J. Orthop. Res. 2019, 37, 449-456. [CrossRef]

26. Segal, Y.; Segal, L.; Blumenfeld-Katzir, T.; Sasson, E.; Poliansky, V.; Loeb, E.; Levy, A.; Alter, A.; Bregman, N. The Effect of Electromagnetic Field Treatment on Recovery from Ischemic Stroke in a Rat Stroke Model: Clinical, Imaging, and Pathological Findings. Stroke Res. Treat. 2016, 2016, 6941946. [CrossRef]

27. Urnukhsaikhan, E.; Mishig-Ochir, T.; Kim, S.C.; Park, J.K.; Seo, Y.K. Neuroprotective Effect of Low Frequency-Pulsed Electromagnetic Fields in Ischemic Stroke. Appl. Biochem. Biotechnol. 2017, 181, 1360-1371. [CrossRef]

28. Lisi, A.; Ledda, M.; Rosola, E.; Pozzi, D.; Emilia, E.D.; Giuliani, L.; Foletti, A.; Modesti, A.; Morris, S.J.; Grimaldi, S. Extremely low frequency electromagnetic field exposure promotes differentiation of pituitary corticotrope-derived AtT20 D16V cells. Bioelectromagnetics 2006, 27, 641-651. [CrossRef]

29. Ross, C.L.; Harrison, B.S. Effect of pulsed electromagnetic field on inflammatory pathway markers in RAW 264.7 murine macrophages. J. Inflamm. Res. 2013, 6, 45-51. [CrossRef]

30. Oda, T.; Koike, T. Magnetic field exposure saves rat cerebellar granule neurons from apoptosis in vitro. Neurosci. Lett. 2004, 365, 83-86. [CrossRef]

31. Li, X.; Xu, H.; Lei, T.; Yang, Y.; Jing, D.; Dai, S.; Luo, P.; Xu, Q. A Pulsed Electromagnetic Field Protects against Glutamate-Induced Excitotoxicity by Modulating the Endocannabinoid System in HT22 Cells. Front. Neurosci. 2017, 11, 42. [CrossRef] [PubMed]

32. Comisso, N.; Del Giudice, E.; De Ninno, A.; Fleischmann, M.; Giuliani, L.; Mengoli, G.; Merlo, F.; Talpo, G. Dynamics of the ion cyclotron resonance effect on amino acids adsorbed at the interfaces. Bioelectromagnetics 2006, 27, 16-25. [CrossRef]

33. Zou, J.; Chen, Y.; Qian, J.; Yang, H. Effect of a low-frequency pulsed electromagnetic field on expression and secretion of IL-1beta and TNF-alpha in nucleus pulposus cells. J. Int. Med. Res. 2017, 45, 462-470. [CrossRef] [PubMed]

34. Liboff, A.R. ION cyclotron resonance: Geomagnetic strategy for living systems? Electromagn. Biol. Med. 2019, 38, 143-148. [CrossRef] [PubMed]

35. Bragin, D.E.; Statom, G.L.; Hagberg, S.; Nemoto, E.M. Increases in microvascular perfusion and tissue oxygenation via pulsed electromagnetic fields in the healthy rat brain. J. Neurosurg. 2015, 122, 1239-1247. [CrossRef] [PubMed]

36. Cheng, Y.; Dai, Y.; Zhu, X.; Xu, H.; Cai, P.; Xia, R.; Mao, L.; Zhao, B.-Q.; Fan, W. Extremely low-frequency electromagnetic fields enhance the proliferation and differentiation of neural progenitor cells cultured from ischemic brains. Neuroreport 2015, 26, 896-902. [CrossRef]

37. Das, S.; Kumar, S.; Jain, S.; Avelev, V.D.; Mathur, R. Exposure to ELF-magnetic field promotes restoration of sensori-motor functions in adult rats with hemisection of thoracic spinal cord. Electromagn. Biol. Med. 2012, 31, 180-194. [CrossRef]

38. Goldshmit, Y.; Banyas, E.; Bens, N.; Yakovchuk, A.; Ruban, A. Blood glutamate scavengers and exercises as an effective neuroprotective treatment in mice with spinal cord injury. J. Neurosurg. Spine 2020, 33, 692-704. [CrossRef]

39. Grace, P.M.; Wang, X.; Strand, K.A.; Baratta, M.V.; Zhang, Y.; Galer, E.L.; Yin, H.; Maier, S.F.; Watkins, L.R. DREADDed microglia in pain: Implications for spinal inflammatory signaling in male rats. Exp. Neurol. 2018, 304, 125-131. [CrossRef] 
40. Chen, Z.; Duan, R.-S.; Quezada, H.C.; Mix, E.; Nennesmo, I.; Adem, A.; Winblad, B.; Zhu, J. Increased microglial activation and astrogliosis after intranasal administration of kainic acid in C57BL/6 mice. J. Neurobiol. 2005, 62, 207-218. [CrossRef]

41. Hodgetts, S.I.; Harvey, A.R. Neurotrophic Factors Used to Treat Spinal Cord Injury. Vitam. Horm. 2017, 104, 405-457. [PubMed]

42. Linker, R.; Gold, R.; Luhder, F. Function of neurotrophic factors beyond the nervous system: Inflammation and autoimmune demyelination. Crit. Rev. Immunol. 2009, 29, 43-68. [CrossRef] [PubMed]

43. Liu, X.-Y.; Chen, C.; Xu, H.-H.; Zhang, Y.-S.; Zhong, L.; Hu, N.; Jia, X.-L.; Wang, Y.-W.; Zhong, K.-H.; Liu, C.; et al. Integrated printed BDNF/collagen/chitosan scaffolds with low temperature extrusion 3D printer accelerated neural regeneration after spinal cord injury. Regen. Biomater. 2021, 8, rbab047. [CrossRef] [PubMed]

44. Bradbury, E.J.; Burnside, E.R. Moving beyond the glial scar for spinal cord repair. Nat. Commun. 2019, 10, 3879. [CrossRef] [PubMed]

45. Oyinbo, C.A. Secondary injury mechanisms in traumatic spinal cord injury: A nugget of this multiply cascade. Acta Neurobiol. Exp. (Wars) 2011, 71, 281-299. [PubMed]

46. Hara, M.; Kobayakawa, K.; Ohkawa, Y.; Kumamaru, H.; Yokota, K.; Saito, T.; Kijima, K.; Yoshizaki, S.; Harimaya, K.; Nakashima, Y.; et al. Interaction of reactive astrocytes with type I collagen induces astrocytic scar formation through the integrin-N-cadherin pathway after spinal cord injury. Nat. Med. 2017, 23, 818-828. [CrossRef]

47. Zamanian, J.L.; Xu, L.; Foo, L.C.; Nouri, N.; Zhou, L.; Giffard, R.G.; Barres, B.A. Genomic analysis of reactive astrogliosis. J. Neurosci. 2012, 32, 6391-6410. [CrossRef]

48. Gris, D.; Marsh, D.R.; Oatway, M.A.; Chen, Y.; Hamilton, E.F.; Dekaban, G.A.; Weaver, L.C. Transient blockade of the CD11d/CD18 integrin reduces secondary damage after spinal cord injury, improving sensory, autonomic, and motor function. J. Neurosci. 2004, 24, 4043-4051. [CrossRef]

49. Cichon, N.; Saluk-Bijak, J.; Miller, E.; Sliwinski, T.; Synowiec, E.; Wigner, P.; Bijak, M. Evaluation of the effects of extremely low frequency electromagnetic field on the levels of some inflammatory cytokines in post-stroke patients. J. Rehabil. Med. 2019, 51, 854-860. [CrossRef]

50. Ross, C.L.; Pettenati, M.J.; Procita, J.; Cathey, L.; George, S.K.; Almeida-Porada, G. Evaluation of Cytotoxic and Genotoxic Effects of Extremely Low-frequency Electromagnetic Field on Mesenchymal Stromal Cells. Glob. Adv. Health Med. 2018, 7 , 2164956118777472. [CrossRef]

51. Cherry, J.D.; Olschowka, J.A.; O'Banion, M.K. Neuroinflammation and M2 microglia: The good, the bad, and the inflamed. J Neuroinflamm. 2014, 11, 98. [CrossRef] [PubMed]

52. Kigerl, K.A.; Gensel, J.C.; Ankeny, D.P.; Alexander, J.K.; Donnelly, D.J.; Popovich, P.G. Identification of two distinct macrophage subsets with divergent effects causing either neurotoxicity or regeneration in the injured mouse spinal cord. J. Neurosci. 2009, 29, 13435-13444. [CrossRef] [PubMed]

53. Greenhalgh, A.D.; David, S. Differences in the phagocytic response of microglia and peripheral macrophages after spinal cord injury and its effects on cell death. J. Neurosci. 2014, 34, 6316-6322. [CrossRef]

54. Tran, A.P.; Warren, P.M.; Silver, J. The Biology of Regeneration Failure and Success After Spinal Cord Injury. Physiol Rev. 2018, 98 , 881-917. [CrossRef] [PubMed]

55. Bilchak, J.N.; Caron, G.; Cote, M.P. Exercise-Induced Plasticity in Signaling Pathways Involved in Motor Recovery after Spinal Cord Injury. Int. J. Mol. Sci. 2021, 22, 4858. [CrossRef] [PubMed]

56. Seanez, I.; Capogrosso, M. Motor improvements enabled by spinal cord stimulation combined with physical training after spinal cord injury: Review of experimental evidence in animals and humans. Bioelectron. Med. 2021, 7, 16. [CrossRef] [PubMed] 